\title{
Mapocho 42k: conectividad de un paisaje ribereño como espacio público memorable ${ }^{1}$
}

\section{Mapocho 42k: connectivity on riparian landscape as memorable public space}

\author{
Sandra Iturriaga del Campo* \\ Mapocho 42k Lab. Facultad de Arquitectura \\ Universidad Católica de Chile, Santiago de Chile \\ director@mapocho42k.cl
}

1- Una versión preliminar de este artículo fue publicada en las Actas de las 1as. Jornadas de Investigación "Ríos urbanos: nuevas perspectivas para el estudio, diseño y gestión de los territorios fluviales". Facultad de Arquitectura y Urbanismo, Universidad Nacional de La Plata / Instituto de Arquitectura y Urbanismo, Universidad Nacional de San Martín, 2 y 3 de noviembre de 2017 ISSN-e 2618-1975

* Arquitecto de la Pontificia Universidad Católica de Chile (PUC), Magister en Arquitectura de la Escuela Técnica Superior de Arquitectura de Barcelona (ETSAB), y actualmente candidata a Doctor en Arquitectura y Estudios Urbanos de la PUC. Desde el año 1997 es docente e investigadora en la Escuela de Arquitectura de la PUC, enfocándose los últimos años en la línea de Proyecto urbano, Patrimonio y Paisaje, vinculado especialmente a entornos fluviales. Desde el 2009 es arquitecto y Directora del proyecto Mapocho 42K. 


\section{Resumen}

El proyecto MAPOCHO 42K surge el año 2009 como parte de una experiencia académica de pregrado al alero de la Universidad Católica de Chile, que luego se desarrolla a lo largo de 8 años como una investigación aplicada, cuyo objetivo se ha encaminado en decantar una propuesta real para la ciudad de Santiago. Su principal punto de partida es recuperar la potencialidad que presentan las riberas del Río Mapocho de conformar un espacio público de escala metropolitana, al modo de una columna vertebral para Santiago en el sentido oriente-poniente, permitiendo consolidarla especial vocación de continuidad geográfica, ambiental y social de este espacio ribereño.

El proyecto propone el acondicionamiento de estas riberas como una gran Promenade geográfica de $42 \mathrm{~km}$ para peatones y ciclistas, que permita conectar todos los estratos sociales y topográficos a través de 11 comunas, y al mismo tiempo, todos los parques existentes o potenciales a lo largo de sus riberas, como parte de un corredor verde ribereño que restituya una continuidad paisajística para la vida de la ciudad, como experiencia cotidiana memorable.

Palabras clave: Promenade ribereña; Espacio público; Paisaje fluvial

\section{Abstract}

The MAPOCHO 42K Project originates in 2009 as part of an undergraduate academic experience under the support of the Pontifical Catholic University of Chile, which later develops as an eight-yearlong applied research, whose main effort has been to become a real proposal for the city of Santiago. Its main starting point is to recover the potentiality that the Mapocho riverbanks still hold to become a Metropolitan scale public space, in the way of a backbone for Santiago from east to west, able to consolidate its special vocation of geographical, environmental and social continuity of the riparian space. The project proposes the recovering of these riverbanks as a large geographical Promenade of $42 \mathrm{~km}$ for pedestrians and cyclist, that allows to connect every social and topographic stratum among 11 communes, and, at the same time, all the existing or potential parks along its path, as part of a riverside green corridor that turn the landscape continuity back to the city life, as an memorable ordinary experience.

Keywords: Riverside promenade; Public space; Riparian landscape 


\section{Introducción:}

\section{El río y sus riberas como paisajememorablede la ciudad}

El río Mapocho es uno de los hechos geográficos más significativos de la ciudad de Santiago, y su condición gravitante en la conformación del paisaje urbano es un desafío que se remonta desdesu fundación. A lo largo de los casi $110 \mathrm{~km}$ que recorre desde su origen en la confluencia de los ríos Molina y San Francisco a los pies de la Cordillera de los Andes, hasta encontrarse con el río Maipo al suroeste de la ciudad en las proximidades de la Cordillera de la Costa, el río Mapocho ha dibujado su marca indeleble en el territorio del valle que atraviesa².

A pesar del importante rol que ha jugado en la historia urbana de Santiago, la relación entre el río Mapocho y la ciudad no ha estado exenta de complejidades y paradojas. A diferencia de otros ríos de llanura como el Sena o el Támesis, su gradiente pronunciada lo caracteriza como un río torrentoso, con variaciones fluviales en diferentes períodos del año y episodios de fuertes crecidas ocasionales. Este hecho, junto con darle una fisonomía inequívoca a su cauce pedregoso,ha implicado un permanente esfuerzo de contención de sus bordes para hacer frente a estas crecidas. ${ }^{3}$ La transformación de sus riberas ha dado lugar a ejemplos notables de una estrecha relación posible entre infraestructura, paisaje y espacio público, la cual perdura en la memoria de la ciudad hasta hoy a partir de dos de sus principales obras de transformación: los tajamaresyla posterior canalización del río. Los primeros, que fueronconstruidos sucesivamente a partir del siglo XVII como un sistema de muros defensivos, dieron origen tempranamente al primer paseo público de Santiago: un recorrido elevado de borde del río que permitía a su paso incorporar la geografía al paisaje urbano ${ }^{4}$, y que junto a

2. Su nacimiento se origina a poca distancia de la ciudad en su salida al valle, cercano a la localidad de lo Barnechea, y en su paso por el valle recibe las aguas de otros afluentes como el estero El Arrayán, el Canal San Carlos, el estero Lampa y el Zanjón de la Aguada.

3. Este carácter torrentoso ha dado lugar a más de 30 inundaciones de gran magnitud a lo largo de la historia urbana de Santiago, lo que determina que hasta hoy el río sea abordado principalmente bajo criterios de riesgo, y las obras que lo comprometen deban contar con la necesaria aprobación de la Dirección de Obras Hidráulicas (DOH).

4. El primer paseo de los Tajamares, llamado "paseo de la Pirámide" que data de 1730, luego sería reemplazado por el más emblemático y definitivo diseñado por el arquitecto Joaquin Toesca en 1792, el cual contaba con casi 4,5m de altura y 1.67 $m$ de ancho, y cuyas ruinas aún se conservan en el Parque de Los Reyes. Sus características han sido bien descritas por: Gó-
Fig.1 Vista de la ciudad de Santiago de Chile con parte del tajamar del Río Mapocho. Fernando Bambrila

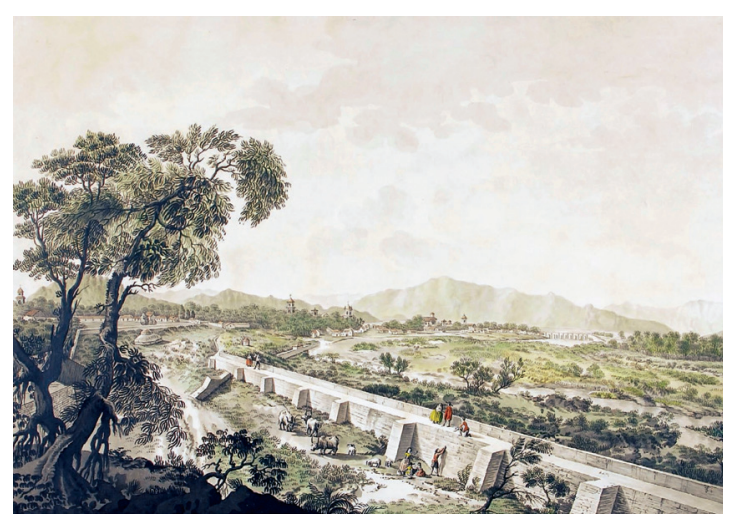

Fuente CMuseo Histórico Nacional

la Alameda que lo acompañaba en sus cercanías, lo convirtieron en un objeto de gran valoración para los viajeros de la época, perdurando en la memoria de la ciudad a partir de este imaginario ${ }^{5}$.(Fig. 1)

Más tarde, a partir de las obras de canalización del río -en los albores del Centenario, se consolidaron en sus márgenes los primeros parques urbanos, que resultan hasta hoy exponentes destacados de recuperación de las riberas como espacio público para la ciudad: el parque Forestal y más tarde el parque Providencia, supieron incorporar una rica relación con el paisaje del río, haciendo eco de su memoria reciente como paseo y espacio urbano privilegiado para la ciudad ${ }^{6}$.

Sin embargo, esta condición ejemplar se contrapuso radicalmente a los criterios que las infraestructuras de vialidad impusieron con el crecimiento de la ciudad. Tanto los ensanches de la vialidad de borde río, su

mez, A; Prado C., Ocaranza F. (2012).Registro arqueológico y contextualización histórica de los tajamares del Río Mapocho. Revista de Historia Regional y local, 4 (8) pp. 275-315.

5. Maria Graham en su Diario de Residencia en Chilelo señala como "un paseo encantador, con larga filas de sauces y una vista espléndida" (Graham,1824:258), y el oficial de marina norteamericano W.S.W. Ruscherberg en su diario de viaje señala que "todo el mundo se pasea en el Tajamar: o bien descansan sobre el parapeto, admirando la hermosura y la grandiosidad del paisaje, u observando a la muchedumbre que pasa". (Ruscherberg,1921:171)

6 . El proceso de transformación de las riberas del Río Mapocho ha sido bien descrito en: Castillo, S. (2014). El Río Mapocho y sus riberas. Santiago de Chile: Ed. U. Alberto Hurtado. 
Fig.2 Río Mapocho en su paso por la ciudad desde Cerro Manquehue

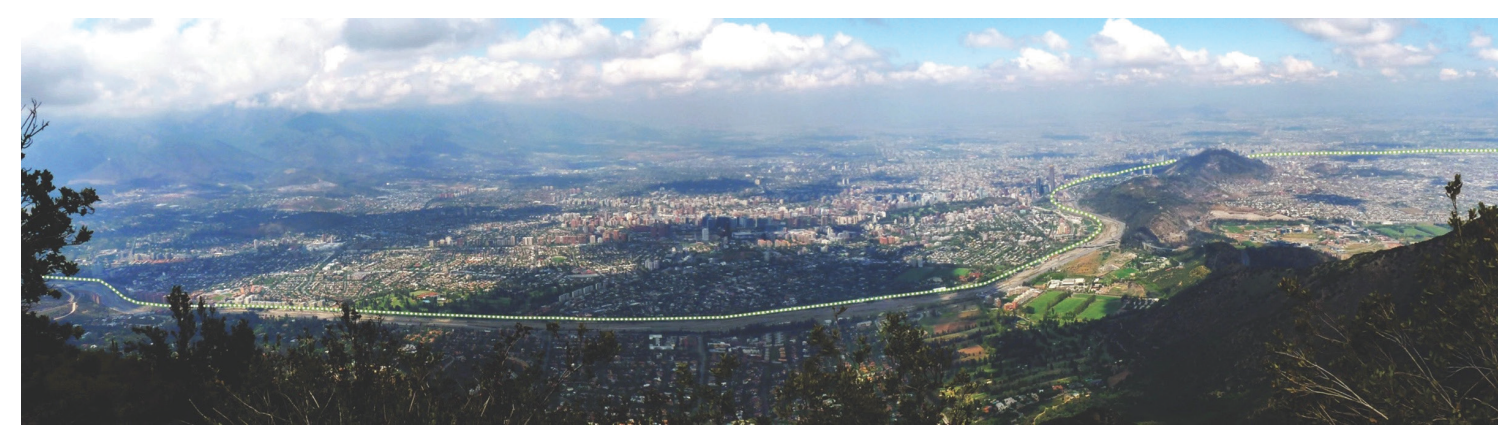

Fuente CMapocho 42K_Lab

interrupción con nudos viales, y la implantación de una autopista de alta velocidad a lo largo de todo su tramo urbano, son expresiones del cambio de paradigma que comenzaron a operar desde la década del 60 y que se acentuó principalmente en los albores del cambio de siglo, hipotecando una nula relación con el río en amplios sectores de la ciudad. Un ejemplo temprano de esta falta de visión es la pérdida del borde río generado por el ensanche de la Avenida Andrés Bello en el tramo central, cortando la continuidad de un paseo arbolado que permitía conectar dos parques centenarios, y más tarde lo impuesto por la Autopista Costanera Norte en todo el margen norte del río, la cual obstruye la posible relación con el río de muchos barrios y comunas aledañas?

Este invasivo proceso de transformación de las riberas del río Mapocho que ha operado en las últimas décadas, se dio paradojalmente en paralelo con un centenar de estudios y proyectos académicos y profesionales del que fue objeto el Río Mapocho, pero que sin embargo no tuvieron el mismo correlato en el espacio físico ${ }^{8}$, permaneciendo el espacio del río en gran medida como un paisajeinvisibilizado para el ciudadano común.

\section{Un proyecto Colectivo desde la Universidad a la Ciudad}

El proyecto MAPOCHO 42K recoge la urgente necesidad de volver a considerar el Río Mapocho comoun espacio

\footnotetext{
7. Resulta inexplicable que aún se sigan planteando obras urbanas basadas sólo en las lógicas de la eficiencia del auto, especialmente si se considera la pérdida de los atributos en torno al borde del río que se hipoteca con estas intervenciones.

8. Sin ir más lejos, en los últimos treinta años - previo al inicio de este proyecto - sobre el río Mapocho se habían desarrollado más de 200 trabajos, entre artículos, tesis, Investigaciones, Seminarios, Planes y Proyectos.
}

públicomemorable para Santiago, entendiendo por ello que el paisaje fluvial que conlleva su presencia en la ciudad,sea un componente esencial capaz de ser integrado a la experiencia cotidiana del ciudadano común.

La propuesta no nace como respuesta profesional a un requerimiento de un mandante o encargo específico, sino más bien como una propuesta académica desarrollada como investigación aplicada, que se formula desde su origen con una fuerte voluntad de articularse como proyecto real para la ciudad. Se origina el año 2009 al alero de un Seminario de pregrado en la Escuela de Arquitectura de la Pontificia Universidad Católica de Chile, el cual se propuso como principal objetivo, comprender un territorio de borde de río de más de 40 km en su paso por la ciudad, tomando como punto de partida el planteamiento del arquitecto Mario Pérez de Arce Lavín. Este planteamiento, plasmado a lo largo de muchos años en un conjunto de artículos de revistas especializadas y de prensa escrita, postulaba la recuperación del Río Mapocho como un Sistema de Parques Integrados para la ciudad, haciendo eco de la impronta que muchos parques centenarios habían otorgado al frente fluvial en su tramo central ${ }^{9}$. Gran parte del esfuerzo de investigación desarrollado durante el Seminario, se volcó en demostrar este planteamientoa partir de un registro exhaustivodel conjunto de hechos y preexistencias delextenso territorio ribereño, entendiendo que la radical contribución de la investigación consistía en

9. Mario Pérez de Arce Lavín (1917-2010), Arquitecto UC 1941, Premio Nacional de Arquitectura 1989. Su visión y conocimientos sobre el río Mapocho y la importancia del mismo para configurar un Sistema de Parques Integrados fue plasmada durante casi 30 años en un sinnúmero de artículos de revistas y prensa escrita. Algunos de los más difundidos son: Pérez de Arce L.,M. (1996). La ciudad y El Río. Revista ARQ, 34, pp.20-21 y Pérez de Arce L.,M. (1997). La Ciudad y la Geografía. Revista ARQ, 37, pp.14-19. 


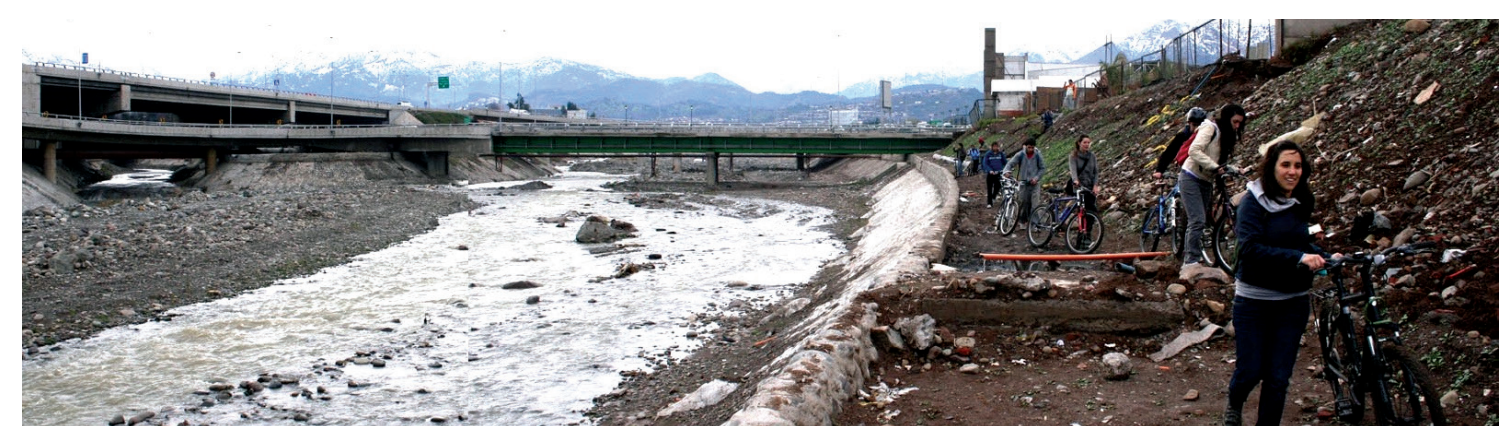

Fuente @Mapocho 42K_Lab,ImagS.Iturriaga

pesquisar y comprender los atributos y fortalezas de este territorio ribereño, antes de hacer esfuerzos anticipados por modificarlo ${ }^{10}$.

El resultado de esta experiencia de investigación se plasmó en la elaboración de un plano de la completitud del río Mapocho urbano y una exposición en agosto del 2010, la cual detonó un interés y resonancia pública. A partir de esto, la propuesta derivó en una investigación aplicada desarrollada a lo largo de casi 8 años por un equipo de arquitectos docentes, con miras a la formulación de un proyecto capaz de ser implementado, insistiendo en su principal componente: recuperar la potencialidad que presentan las riberas del río Mapocho de conformar un espacio público de escala metropolitana, al modo de una gran Promenade ribereña a lo largo de $42 \mathrm{~K}$ en su paso por la ciudad, que permita conectarla social y geográficamente (Fig. 2).

Desde sus inicios, la investigación se postuló para su concreción,la necesidad de poder articular desde la Academia la condición pública de un proyecto urbano de esta escala. Estoimplicó,por un lado, potenciar una fuerte vocación de proyecto colectivo, con la capacidad de convocar a un conjunto importante de actores relacionados con la gestión del territorio ribereño -tanto públicos como privados, especialmente de las 11 comunas comprometidas en sus márgenes. trasladandoen gran medida la figura del arquitecto como autor, a la decoautor de un proyecto común, y por otro lado, posibilitar que la capacidad de articulación entre los distintos actores se diera principalmentea partir de los propios

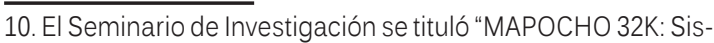
tema de Parques Integrados en las Riberas del Rio Mapocho", el cual fue realizado por la profesora Sandra Iturriaga y Mario Pérez de Arce L. como profesor invitado, y la colaboración de Francisco Quintana. mecanismos de representación de la propuesta. Se tratará de mostrar lo más fielmente posible,el itinerario que ha llevado a este proyecto desde su condición de investigación aplicada -centrada principalmente en la representación cartográfica del río como hecho urbano destacado-, hasta su actual implementación por tramos, permitiendo la continuidad de esta iniciativa en el tiempo a partir de tres premisas fundamentales.

\section{Del Territorio al Mapa: Itinerario de una propuesta}

Si consideramos que en la noción de paisaje, a diferencia del simple entorno o 'medio ambiente', está implícita la valoración de un determinado territorio por parte de quien lo experimenta (Maderuelo, 2005), en el caso del río Mapocho -a pesar de la existencia de parques centenarios y del logro por el saneamiento de sus aguas- esta valoración no era para nada evidente. En los inicios de la investigación durante el curso de pregrado, la primera premisa que se postuló por tanto, consistió en confrontar la posibilidad real de recorrer las riberas del río Mapocho a lo largo de todo su tramo urbano, en una experiencia directa con este paisaje fluvial, bajo el supuesto que la reticencia por reconocer al río Mapocho como un paisaje vivo y memorable dentro de la ciudad, se debía en parte a la dificultad de poder recorrerlo, quedando esta experiencia relegada sólo a sectores puntuales.

En contraste con la continuidad conseguida por la vialidad expresa en su ribera norte, el potencial de un recorrido continuo a lo largo de la ribera sur, que permitiera integrar el paisaje de río al interior de la ciudad, era un valor a constatar. Para ello se realizó un itinerario en bicicleta a lo largo de todo el tramo urbano, que a poco de iniciarse significó tener que sortear un sinnúmero de situaciones que interrumpían sus bordes o lo 
Fig.4 Plano Santiago. Juan Herbage 1841

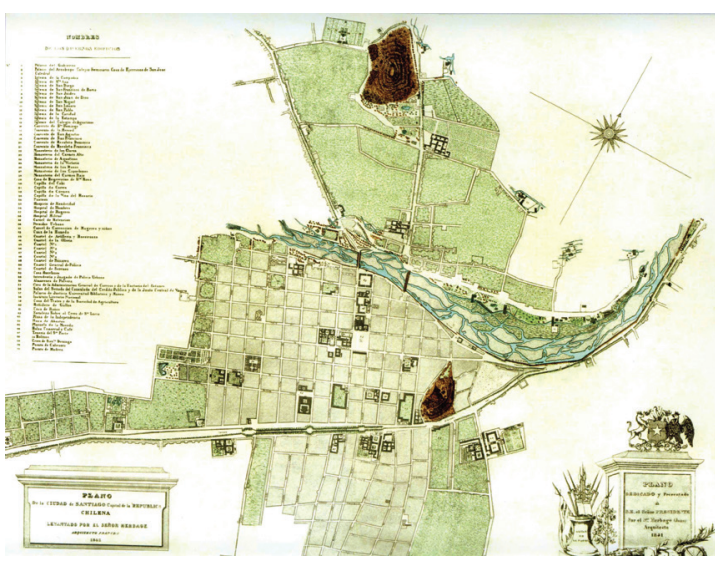

Fuente @ Colección Biblioteca Nacional de Chile

hacían francamente inaccesible: cruces de autopistas, sitios eriazos, asentamientos informales, ensanches de vialidad, y un conjunto de barreras existentes aún en sectores consolidados(Fig. 3).

Esta evidente fragmentación de las riberas, producto de un proceso de crecimiento desregulado de la ciudad y de la división administrativa del territorio ribereño, es algo que lejos de ser una excepción, pareciera un lugar común de un conjunto de otros casos del hemisferio sur que se revisaron en paralelo, evidenciando que la historia de los ríos urbanos pareciera seguir un mismo sino.

La segunda premisa,consistió en poder hacer del registro y la representación, la principal herramienta para visibilizar -que a pesar de lo anterior-el Río Mapocho aún presentaba un gran potencial de configurar un espacio público vertebrador de la ciudad, especialmente a partir de su ribera sur. Fue significativo comprobar que, a pesar de la gran cantidad de estudios y proyectos sobre el río Mapocho realizados a la fecha, no existía un registro exhaustivo del río y sus riberas que representara en completitud su tramo urbano a partir del levantamiento de sus preexistencias ${ }^{11}$. Tomando como base la noción de cartografía propuesta por Guallart, se trataba por tanto, de poder representar el territorio ribereño y sus principales elementos como una manera de comprender y

11. Elprimer recorrido a lo largo del Río Mapocho se realizó en Agosto de 2009 en el marco del curso de pregrado, siendo muy relevante para el trabajo de levantamiento cartográfico posterior, ya que sentó las base de un conocimiento directo de sus potencialidades. Este primer recorrido de verificación contó con la participación de 12 estudiantes, con los cuales se realizó un trabajo de investigación fundamentalmente en base al levantamiento directo del río. mostrar sus atributos, antes de realizar cualquier esfuerzo por modificarlo, entendiendo que "representar un territorio es comenzar a transformarlo".(Guallart, 2001:53)

No es casual que la consideración del río como un fenómeno vivo y vivido resulte tan elocuente en los planos y cartografías de Santiago durante sus primeros siglos de historia urbana, en contraposición a su actual representación: en el plano de Amédeé Frezierde 1716 por ejemplo ${ }^{12}$ , el río aparece reflejado como un fenómeno que al menos equipara en interés al de la trama urbana, o en el plano de Juan Herbage de $1841^{13}$, la permanente negociación con sus riberas para hacer frente a las crecidas se vuelve, una vez representada, una cualidad urbana (Fig.4).

Del mismo modo, uno de los ejemplos más notables del conocimiento, y con ello la identidadde un territorio instalada a partir de la representación y el registro del mismo, lo constituye el esfuerzo realizado a mediados del siglo XIXpor el naturalista francés Claudio Gay. Las cartografías y dibujos que forman parte de suAtlas de la Historia Física y política de Chile dan cabida tanto a una representación cartográfica a escala del territorio como a la de un extenso y preciso registro de especies vegetales, animales y otros(Gay,1865).Junto con representar la realidad, sus mapas se anticipan a ella, transformándose en modelo y guía para las acciones futuras del joven estado republicano ${ }^{14}$.

Esto nos llevó a pensar en la escala adecuada para la cartografía del río, como uno de los principales componentes metodológicos para su verificación, considerando que en el plano Intercomunal de Santiago -que es la base donde se sustenta su planificación,el río Mapocho y sus riberas se reducen a una situación esquemática, casi con la misma valoración que la de una vialidad ${ }^{15}$.

La principal tarea consistió, por tanto, en la elaboración de una cartografía exhaustiva y detallada a escala 1:5.000 ${ }^{16}$ durante un período de casi un año, con el fin

12. Plan de la Ville de Santiago, Amédeé Frezier,1716.

13. Plano de la ciudad de Juan Herbage,1841.

14. La primera edición del Atlas data de 1854, y consideró un colosal esfuerzo que le tomó algo más de 20 años concretarlo, y comprendía 2 volúmenes con 315 láminas coloreadas a mano.

15. En el plano Intercomunal de Santiago, el ancho existente del río en sus áreas centrales de $42 \mathrm{~m}$ canalizado, se reduce a una medida de $2 \mathrm{~mm}$.

16. Los atributos de la representación cartográfica a Escala 1:5.000 forman parte de la reflexión metodológica que ha llevado adelante el equipo de investigadores José Rosas, WrenS- 
Fig.5 Cartografía Río Mapocho Escala 1.5.000

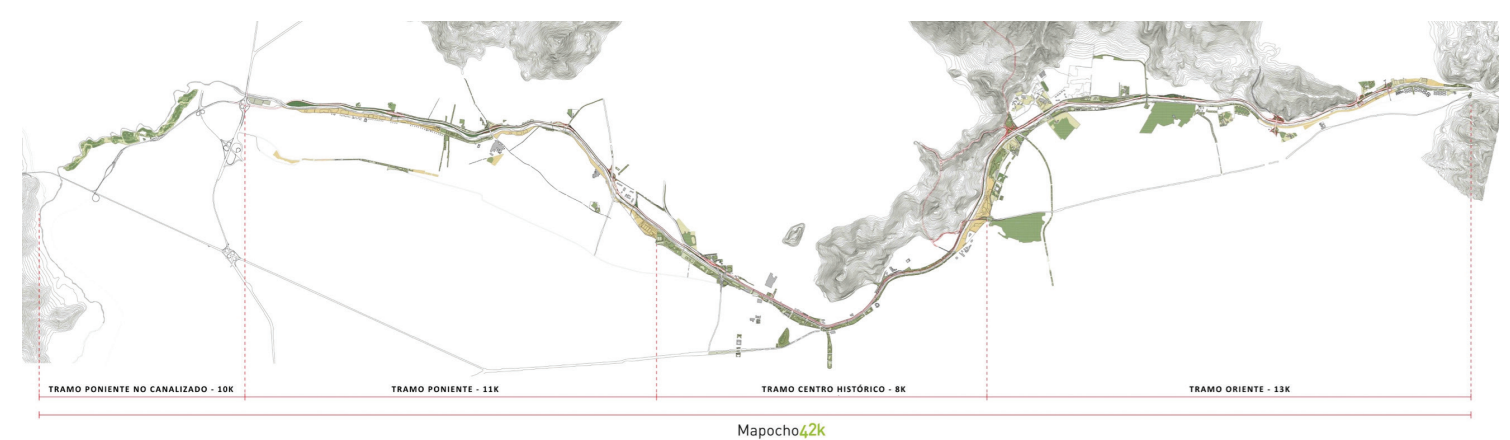

Fuente @Mapocho 42K_Lab

de construir un registro propositivo del territorio abordado a partir del total de sus preexistencias ${ }^{17}$, tomando como punto de partida loexpresado por el urbanista español Manuel de Solá Morales en relación al registro del territorio de Catalunya,

y que operó como la base para sus transformaciones: "dibujar es seleccionar, seleccionar es interpretar, interpretar es proponer" (Solá Morales,1981:4)

Esta premisa,resultó central en la elaboración del plano del río Mapocho, y constituyó la base sobre la cual se elaboró en los dos años posteriores el trabajo profesional que daría pie a una estrategia general como a los diseños de tramos específicos, actuando como una memoria persistente de que completar el acondicionamiento del río y sus riberas era sólo una cuestión de tiempo (Fig.5)

\section{Recuperar el borde río como una Promenade geográfica}

A partir de este registro cartográfico, uno de los principales componentes que se evidenció como atributo importante del río fue su arbolado, el cual formaba par-

trabucchi y Germán Hidalgo de la Universidad Católica, a partir de la investigación sobre la ciudad de Santiago de 1910, y que más recientemente ha sido referenciada en el libro:Santiago 1910. El canon republicano y la distancia cinco mil (Rosas, Parcerisa, 2016).

17. Se elaboró un catastro inicial de 5 meses, con un esfuerzo permanente de verificación de diferentes fuentes planimétricas con la realidad del lugar, con el propósito de incorporar todas aquellas preexistencias comprendidas a lo largo del río Mapocho en su tramo urbano desde la Plaza San Enrique hasta Pudahuel: parques existentes, calles principales, sitios eriazos, edificación relevante, arborización, caracterización de suelos principales, entre otros.Posterior a ello se dedicaron 8 meses a revisar, elaborar y editar este plano,1:5.000 y de 1,8 $\mathrm{m} \times 7 \mathrm{~m}$. te tanto de las áreas consolidadas de parques, como de aquellos sitios eriazos al Oriente y al Poniente, que hasta fines de los 90 aún formaban parte de un cauce mayor aún no canalizado. Como contraparte, a pesar de la existencia de un conjunto de parques de borde río existentes y potenciales, la continuidad entre estos territorios era un atributo escaso. Ambas condiciones, la de potenciar un corredor verde ribereño -a partir del arbolado ya existente-, y la de restituir la continuidad del borderío -como condición para su apropiación como paisaje vivo de la ciudad, sentaron las principales bases de la propuesta:

Elproyecto Mapocho 42K formula elacondicionamiento de las riberas del Río Mapocho, principalmente la ribera sur, como un gran paseo de uso público y esparcimiento, una Promenade metropolitana que atraviesa42 km en su paso por la ciudad, permitiendo restituir una continuidad paisajistica y geográfica desde la entrada al valle del Río Mapocho a los pies del macizo cordillerano, hasta el inicio de las localidades rurales al poniente, próximos a la cordillera de la costa. (Fig.6)

Junto con restituir esta conectividad geográfica y paisajística, la propuesta busca conectar 11 comunas ribereñas de diferentes estratos sociales y topográficos, consolidando una vocación de continuidad a lo largo del Río en oposición a la fragmentada división administrativa de sus riberas ${ }^{18}$. Se postula que la conectividad geográfica y paisajística del Río Mapocho, se transforme en el principal atributo de equidad territorial para la ciudad.

18. Existe un total de 11 comunas colindantes con el Río Mapocho en su tramo urbano, y 16 comunas en total hasta su encuentro con el Río Maipo, presentándose los mismos efectos de la fragmentación comunal que se evidencia en el resto del territorio metropolitano. 


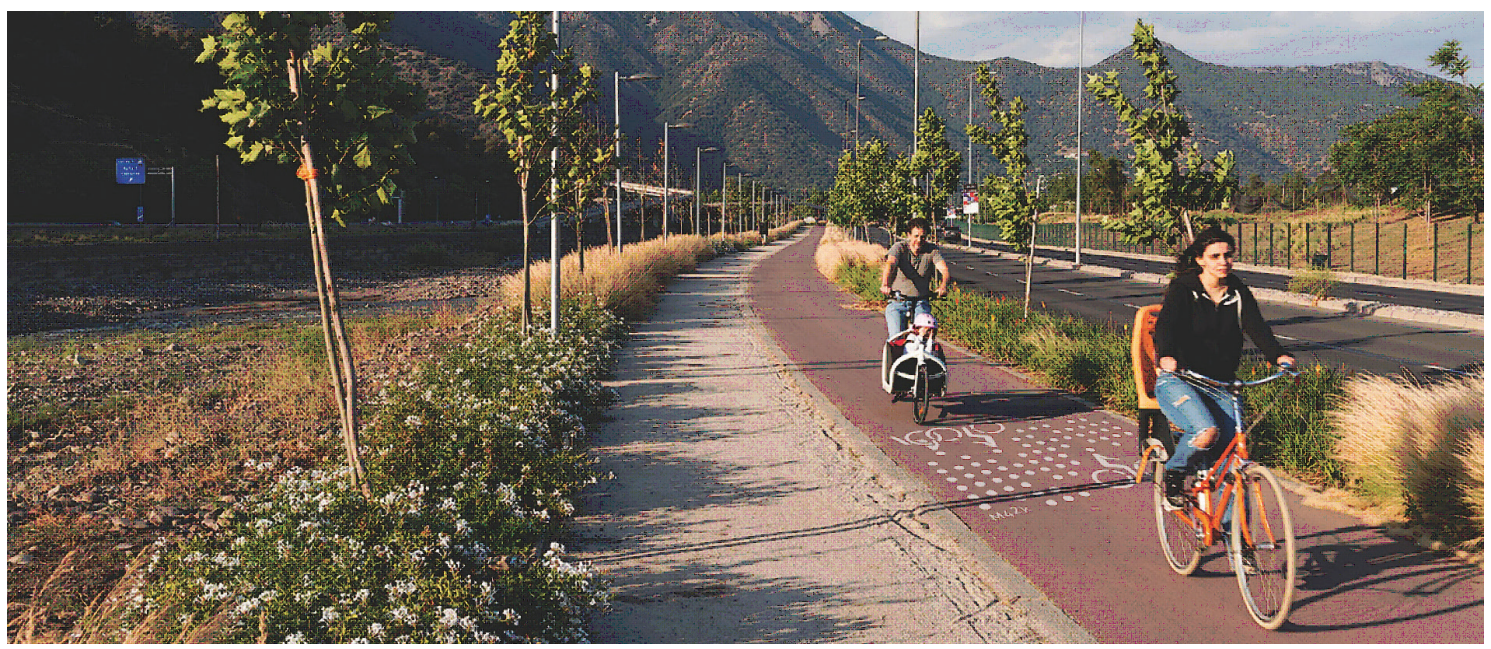

Fuente @Mapocho 42K_Lab, Imag C. Correa

Fig.7Propuesta Cicloparque Mapocho 42K como corredor ambiental y paisajístico

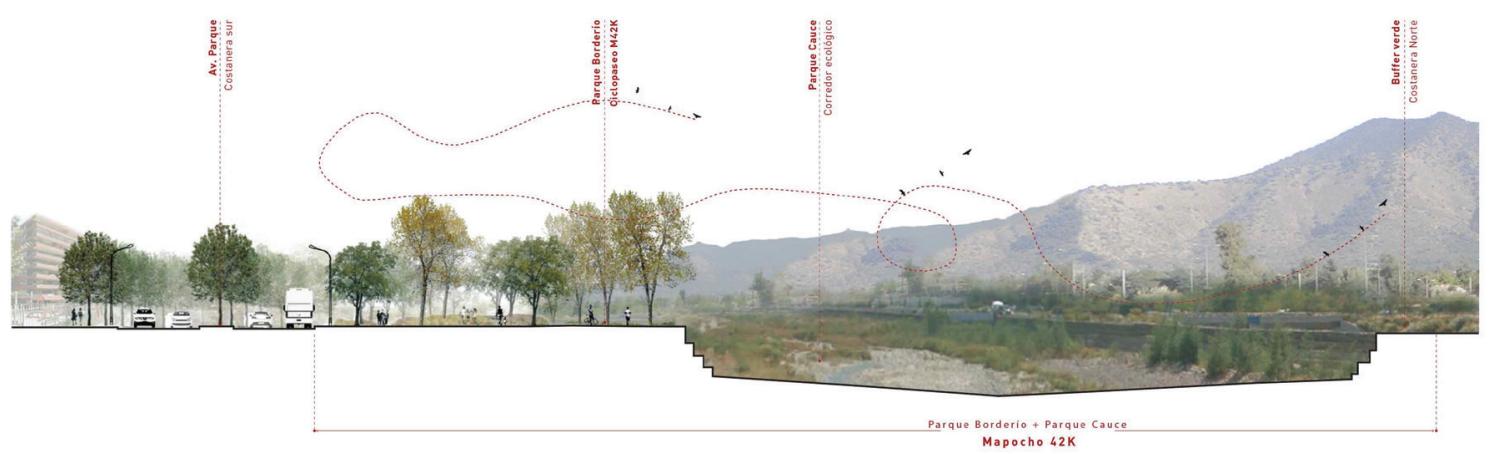

Fuente CMapocho 42K_Lab

Esta conectividad geográfica y social se propone a partir de la implementación de una Vía Verde o Ciclo parque ${ }^{19}$, capaz de acoger tanto a peatones y ciclistas -sea de paseo, de deporte o de traslado, a la sombra de un recorrido de borderío que potencie su condición de balcón urbano y geográficocomo principal atributo para la ciudad.Este corredor verde permite conectar al mismo tiempo todos los parques existentes o potenciales a lo largo del borderío, relacionándolos con otros parques o cerros cercanos, colaborando

19. El concepto de Cicloparque se relaciona con la noción de Greenway, o Vías Verdes, definidas como "infraestructuras autónomas destinadas al tráfico no motorizado, ya sea de peatones o ciclistas, personas con movilidad reducida, patinadores, etc. desarrolladas en un marco integrado que valore el medio ambiente y la calidad de vida".Guía de buenas prácticas de Vías Verdes en Europa (2000) Bélgica. en consolidar una matriz geográfica y ecológica para Santiago, como principal componente de su desarrollo urbano (Fig. 7).

La propuesta no apunta por tanto a ser una solución unívocaatemas de movilidad o "áreas verdes"en relación a la consolidación de este borde fluvial-aun cuandopuede ser un aporte frente a la tradicional segmentación con los cuales se los planifica- sino que propone integrar simultáneamentea partir de esta Promenade ribereña, valores ambientales, urbanos, y paisajísticos(Fig.8).

Por un lado, haciendo eco de las palabras del ecólogo Richard Fordman, quien postula que la ciudad debiera ser pensada para personas entre los 7 y los 70 años(Fordman,1986), este corredor ribereño se propone 
Fig.8 Propuesta Cicloparque Mapocho 42K como corredor ambiental y paisajístico
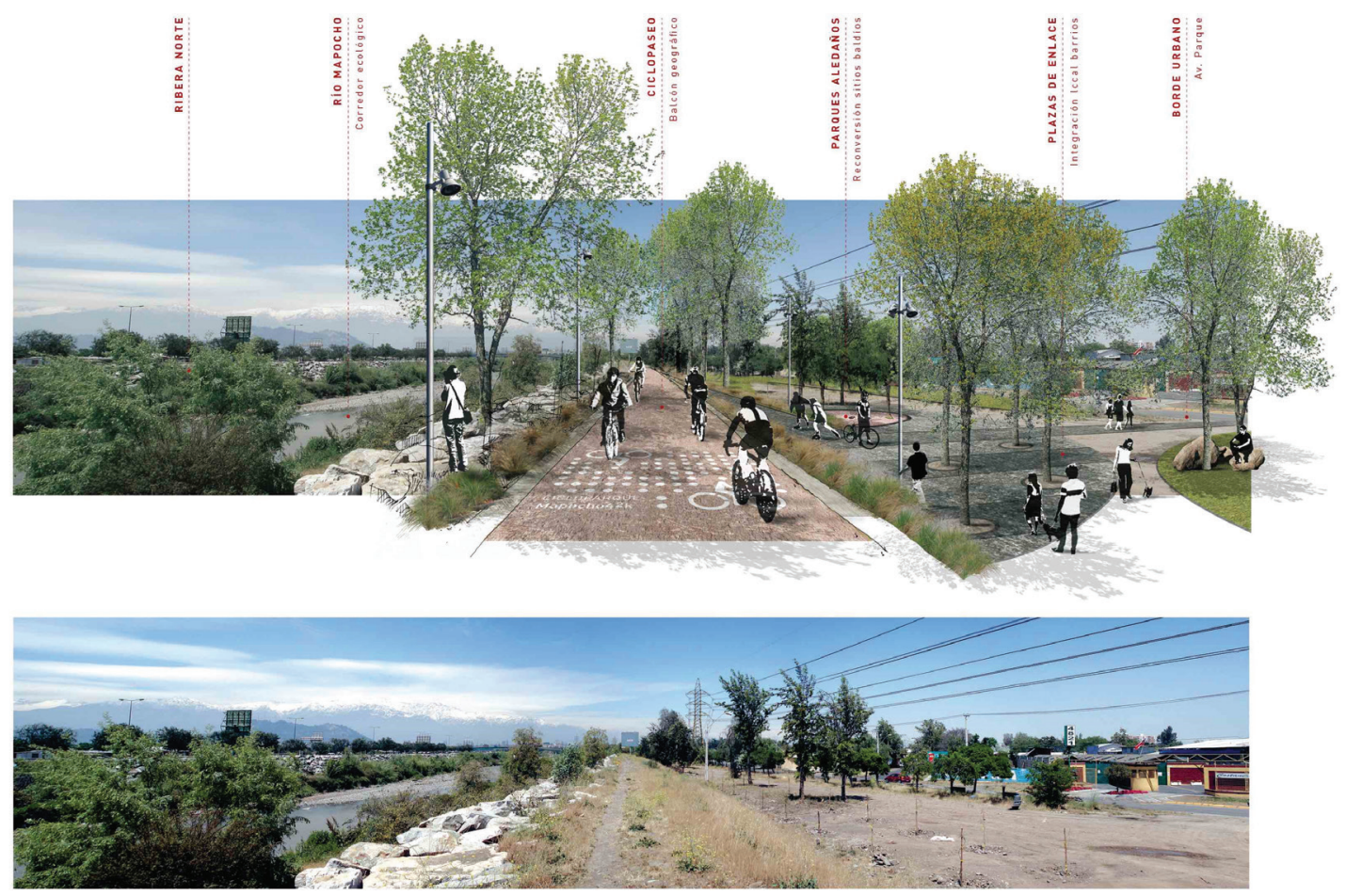

Fuente CMapocho 42K_Lab

comouna vía verde de movilidad sustentable, que a partir de sus componentes es capaz de acoger a distintos tipos de usuarios en un espacio de seguridad y valoracióndel entono circundante.Por otro lado, se traduce en una experiencia directa con el espacio del río a partir de poder recorrerlo, desde el cual poder percibir sus atributos ambientales y cambios estacionales a lo largo del año -que transforman su cauce en la extensión de un único gran parque-, y donde se integra el espacio circundante como una ventana a la geografía, como parte identitaria de su memoria en la ciudad.

Muy distinto es que los $\mathrm{m} 2$ de área verde por habitante -al que se alude como un indicador propuesto por la OMS para medir la calidad de vida en las ciudades- sea un conjunto de retazos que la ciudad no supo aprovechar, a que constituyan en conjunto un gran parque integrado a la espacialidad del río, como un espacio público de dimensión geográfica con una situación inmejorable para la ciudad ${ }^{20}$ (fig.9).

20. La normativa urbana chilena favorece la proliferación de áreas verdes de pequeño tamaño, ya que se basa en un \% destinado a este fin y no establece un tamaño mínimo, existiendo en Santiago un 91\% de áreas verdes separadas, con menos de 0,5 há(Reyes, Figueroa 2010).

\section{Conectar un paisaje ribereño: consolidación de un bor- de fluvial de $42 \mathrm{~K}$}

La primera etapa de desarrollo delproyecto con posterioridad a la fase de levantamiento -desde Septiembre del 2012 en adelante- consideró la elaboración de un Plan estratégico para el total del Río Mapocho, que permitiera verificar la factibilidad de poder implementar este corredor verde continuo a lo largo de $42 \mathrm{~km}$ por la ribera sur, con una evaluación de sus aspectos normativos, legales y urbanos.

Al mismo tiempo se trataba de diagnosticar las situaciones críticasy puntos de conflicto donde este paseo de borde río requería de una estrategia de intervención mayor que restituyera la conectividad, especialmente en aquellos sectores ligados a nodos de vialidad urbana que requerían una importante gestión por parte de las autoridades, arriesgando de lo contrario una fragmentación del espacio ribereño.

En esta etapa se hizo necesario hacer converger en torno a la propuesta, al conjunto de actores públicos bajo la figura de un comité estratégico, a partir del cual se logró un acuerdo que permitiera pasar -simbólica y 
Fig.9 Cicloparque Mapocho 42Kejecutado en Tramo PonienteQuinta Normal_2015

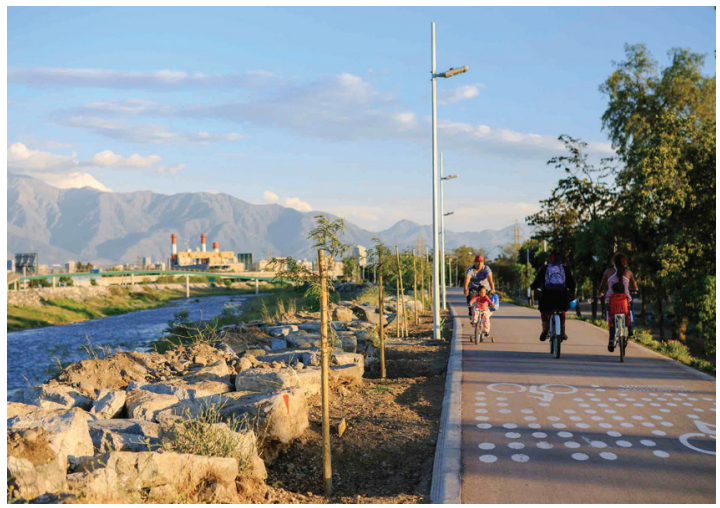

Fuente @Mapocho 42K, imag F. Croxatto

funcionalmente- de un territorio fragmentado a un espacio colectivo ${ }^{21}$. Al final de esta etapa, el Ministerio de Vivienda comprometió recursos para su ejecución, definiéndose a partir del estudio realizado un conjunto de tramos a implementar en una primera etapa de ejecución ${ }^{22}$

La segunda etapa -desarrollada desde inicios del 2013 hasta el 2016-, contempló la propuesta de diseño e ingeniería de detalles del Ciclo parque Mapocho 42K,a partir de una selección de tramos que permitieranimplementar y restituir una primera conectividad de casi $20 \mathrm{~km}$, conectando el Parque Bicentenario en la comuna de Vitacura, con el Parque Mapocho Poniente en la comuna de Cerro Navia.

Durante esta etapa se delinearon los criterios de diseño comunes para todo el recorrido, estableciéndose dos conceptos claves para la definición del proyecto:

Por un lado, la estrategia de diseño consideró una "matriz abierta", esto es, en base a una paleta de componentes constructivos y espaciales -consensuados con los municipios-,que permitieran establecer una identidad común y un carácter unitario a lo largo del recorrido, y al mismo tiempo, que permitieran ser implementa-

21. Con el fin de fomentar esta integración de forma sostenida se implementó una plataforma web interactiva que ha resultado una eficaz herramienta de interacción y socialización del proyecto, especialmente con la ciudadanía.

22. El proyecto ha sido fruto de la articulación de distintos actores desde sus inicios, donde un Convenio de Colaboración con el Ministerio de Vivienda le ha permitido llevar adelante las obras de ejecución desde el año 2012 a la fecha, siendo los Municipios una contraparte.
Fig.10 Cicloparque Mapocho 42K ejecutado en Tramo Central de Providencia_2016

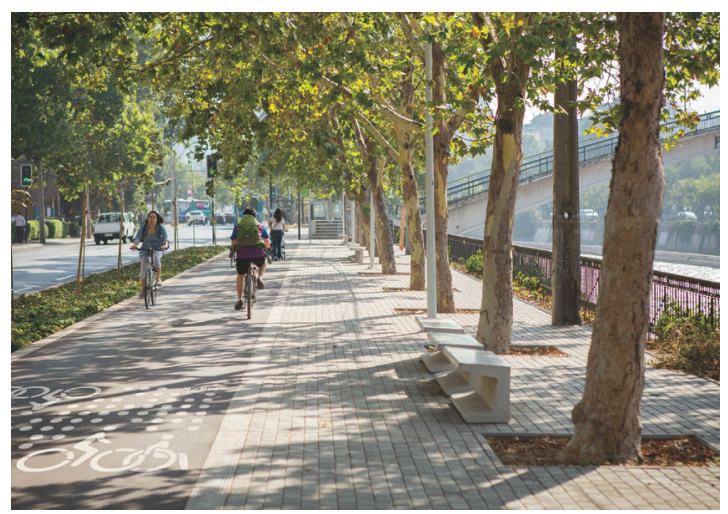

Fuente CMapocho42K_Lab, Imag F. Croxatto

dos por los distintos municipios como un kit de piezas fácilmente aplicable ${ }^{23}$.

Por otro lado, la propuesta considera aprovechar la posición de balcón urbano abierto a la geografía como un atributo esencial a lo largo de toda la ribera del Río Mapocho, desde el cual consolidarel principal componente del recorrido: un corredor arbolado continuo, bajo cuya sombra se sitúa tanto el paseo peatonal como la senda ciclable, y que se presenta como el elemento identitario y estructural del borde río(Fig.10).Al igual que ocurría con los antiguos tajamares, esta pieza estructurante y unitaria del recorrido, se propone al modo de una infraestructura que ordena y mide el resto de las intervenciones de borde, siendo el principal conector del Sistema de parques que se sitúan a lo largo de la ribera del Río Mapocho ${ }^{24}$.

La propuesta consideraal mismo tiempo, una faja de intervención en un espesor variable -entre 10 y 25 m-, donde este paseo principal se va ritmandocon diversas situaciones de descanso, zonas de juego o zonas de articulación con la trama urbana.Las áreas de vegetación complementarias al corredor principal consideran especies que se adaptan a nuestro clima semiárido, con bajo requerimiento hídrico, destacando las texturas y colores que potencian la condición cinética de la vía verde.

23. Los componentes consideran tanto elementos constructivos como espaciales, abarcando desde lluminación, pavimentación, mobiliario urbano y vegetación.

24. paseo ciclable considera un ancho constante de $3 \mathrm{~m}$, de modo de incorporar una condición inclusiva para personas con movilidad reducida. Su materialidad en carpeta asfáltica de color y una solera de hormigón diseñada para los rodados garantiza la unidad a lo largo de los 42K. 
Fig.11 Propuesta Cicloparque Mapocho 42K Tramo Poniente Cerro Navia

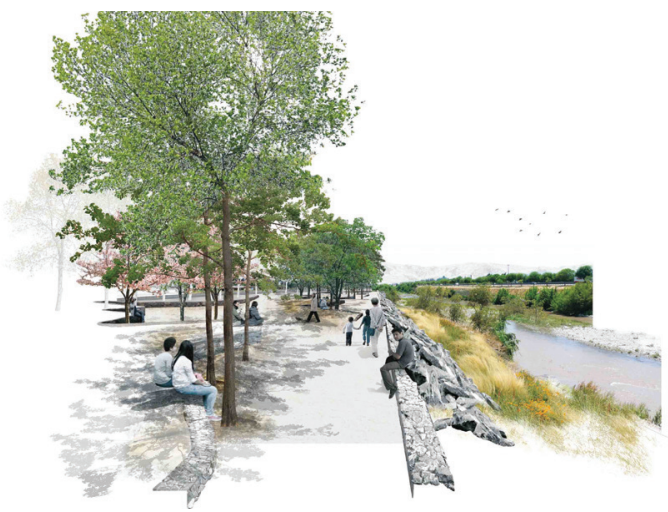

Fuente CMapocho42K_Lab

Por último, la propuestaplantea una tercera etapa -actualmente en elaboración- queconsidera el desarrollo de un conjunto de áreas y parques de borde río tanto al Oriente como al Poniente, que junto con integrarse al sistema de parques borde río y a la experiencia completa del recorrido, posibilitan una mayor interacción con el Río a partir de su relación de proximidad con el cauce. Esta relación de proximidad -tanto perceptual como física-, permite ampliar el espectro de oportunidades en la relación con el Río Mapocho, ofreciendo nuevos modos de apropiación con su entorno inmediato (Fig.11). Sin embargo, junto con ello, la
Fig.12 Río Mapocho Aguas Abajo en localidad El Monte, 2017

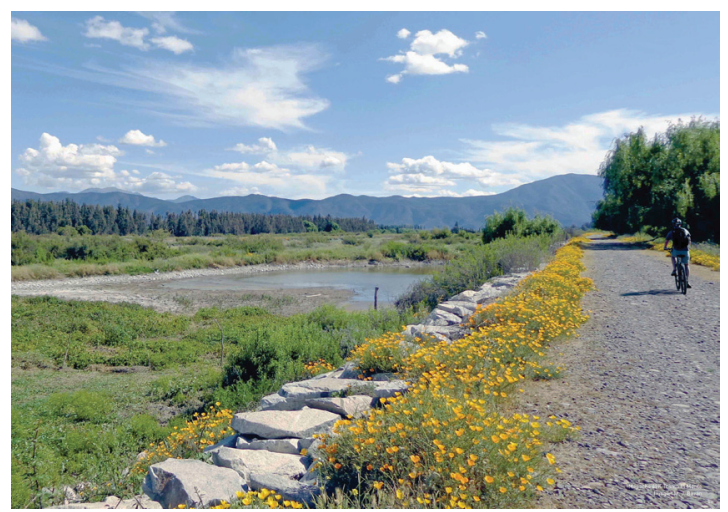

Fuente @Mapocho42K_Lab, ImagM.J.Bazán

consideración del Río como un elemento de gran valor ambiental y ecológico, exige para su mantención en el tiempo nuevos alcances desde la gestión pública, dado que una mayor proximidadimplica muchas veces mayores niveles de erosión.

Luego de ocho años de continuidad de esta investigación aplicada, es posible pensar que, a este primer esfuerzo de consolidación delRío Mapocho como un gran conector geográfico y social en su tramo urbano, le seguirá en breve plazo la puesta en valor de este corredor verde en su total extensión hasta el Río Maipo (Fig.12)

\section{Bibliografía}

Castillo, S. (2014). El Río Mapocho y sus riberas. Santiago de Chile: U. Alberto Hurtado

Claudio Gay, C. (2010(1865)). Atlas de la Historia Física y Política de Chile. Tomos I y II. Santiago de Chile: LOM

Forman,R., Gordon, M. (1986). Landscape Ecology. Nueva York: Ed. John Wiley \& Sons

Gómez, A; Prado C., Ocaranza F. (2012). Registro arqueológico y contextualización histórica de los tajamares del Río Mapocho. Revista de Historia Regional y local, 4 (8) pp. 275-315.

Graham, M. (1824).Diario de Residencia en Chile. Madrid: América. pp. 258.

Guallart, V. (2001). Cartografía. En: AAVV, Metápolis. Diccionario de Arquitectura Avanzada. Barcelona: Actar. pp.53

Maderuelo, J. (2005). El paisaje, génesis de un concepto. Madrid: Abada Editores

Pérez de Arce L.,M. (1996). La ciudad y El Río. Revista ARQ, 34, pp. 20-21

Pérez de Arce L.,M. (1997). La Ciudad y la Geografía. Revista ARQ, 37, pp. 14-19

Ruscherberger, W.S.W. (1921).Noticias de Chile (1831-1832), por un oficial de Marinade los EEUU de América. Revista Chilena de Historia y Geografía, XI (42), pp.170-185.

Solá Morales, M., Parcerisa,J.(1981).La forma de un País. Revista Quadernsd'Arquitectura i Urbanisme,1, pp.4-8

Reyes, S., Figueroa, I.M. (2010).Distribución, superficie y accesibilidad de las áreas verdes en Santiago de Chile, Revista EURE, 36 (109)

Rosas, J., Parcerisa, J.(2016). Santiago 1910. El canon republicano y la distancia cinco mil. Santiago de Chile: Ediciones UC 